\title{
A Performance Evaluation of Commercial Fibrinogen Reference Preparations and Assays for Clauss and PT-derived Fibrinogen
}

\author{
I. J. Mackie', A. S. Lawrie', S. Kitchen², P. J. Gaffney 3, D. Howarth 4, G. D. O. Lowe ${ }^{5}$, \\ J. Martin ${ }^{4}$, G. Purdy' , P. Rigsby ${ }^{3}$, A. Rumley ${ }^{5}$
}

'The Haematology Department, University College London, London; 2 Sheffield Haemophilia and Thrombosis Centre, Royal Hallamshire Hospital, Sheffield:

${ }^{3}$ National Institute for Biological Standards and Control, Potters Bar; ${ }^{4}$ MRC Epidemiology \& Medical Care Unit, Wolfson Institute of Preventative Medicine, London; ${ }^{5}$ Department of Medicine, University of Glasgow, UK

\section{Keywords}

Fibrinogen assays, coagulation analysers, Clauss reagents, thromboplastin reagent, reference plasmas

\section{Summary}

The wide availability of fibrinogen estimations based on the prothrombin time (PT-Fg) has caused concern about the variability and clinical utility of fibrinogen assays. In a multi-centre study, we investigated fibrinogen assays using various reagents and analysers. Clauss assays generally gave good agreement, although one reagent gave $15-30 \%$ higher values in DIC and thrombolysis. Two commercial reference preparations had much lower potencies than the manufacturers declared, and plasma turbidity influenced parallelism in some Clauss assays. PT-Fg assays gave higher values than Clauss and showed calibrant dependent effects, the degree of disparity correlating with calibrant and test sample turbidity. Analyser and thromboplastin dependent differences were noted. The relationship between Clauss and PT-Fg assays was sigmoid, and the plateau of maximal PT-Fg differed by about $2 \mathrm{~g} / \mathrm{l}$ between reagents. ELISA and immunonephelometric assays correlated well, but with a high degree of scatter. Antigen levels were higher than Clauss, but slightly lower than PT-Fg assays, which appeared to be influenced by degraded fibrinogen. Clauss assays are generally reproducible between centres, analysers and reagents, but PT-Fg assays are not reliable in clinical settings.

\section{Introduction}

Fibrinogen assays are performed in the investigation of haemorrhage, monitoring of thrombolytic therapy and in risk factor screening for arterial disease. The Clauss fibrinogen assay (1), based on the thrombin clotting time, is the most frequently used technique, but varies greatly in the source and composition of reagents, as well as assay protocol. In developed countries, automated methods, based on photooptical principles, are most frequently used. Most assays are designed for measuring low fibrinogen levels and may have different sensitivity or accuracy for elevated fibrinogen.

Correspondence to: Dr. I. J. Mackie, Haematology Department, University College London, 48 Riding House Street W1N 8AA, UK - Tel. +44 (0)20 7679 9686; Fax: +44 (0)20 7679 9684; E-mail: i.mackie@ucl.ac.uk
The choice of commercial calibrant plasma is clearly important and it has been recommended that manufacturers calibrate against the International Standard (IS) preparation (2). The 1st IS for plasma fibrinogen has been available for many years (3), but some manufacturers of fibrinogen 'standards' have been slow to utilise it, and apparently calibrate against "in house" plasma pools or the College of American Pathologists standard. The 1st IS was calibrated primarily by clottable protein assays rather than by the Clauss technique (since any primary standard must be calibrated by a direct or absolute measure), and a change to the IS could cause a significant shift in calibration values, requiring new reference ranges.

The numerous types of coagulation analysers introduce further variables; many offer a fibrinogen estimation based on the change in light scattering or optical density during the prothrombin time (the PTderived fibrinogen, PT-Fg). This method rapidly provides a fibrinogen value and is used in approximately $50 \%$ of UK Haematology laboratories (4). However, some PT-Fg methods give higher fibrinogen values than the Clauss technique $(2,5-8)$, although the discrepancy is not consistent over a range of fibrinogen levels, and may depend on the clinical defect as well as the thromboplastin reagent used $(7,8)$. The PTFg method gives apparently normal fibrinogen levels in certain clinical states, where Clauss levels are reduced, e.g. dysfibrinogenaemia and thrombolytic therapy. Despite these observations, some groups demonstrated good comparability between Clauss and particular PT-Fg assays (9-10), although the latter stated that precision was inadequate in PT-Fg assays where the PT was prolonged (e.g. due to anticoagulants) and if the fibrinogen level was high.

The purpose of this study was therefore to evaluate the most frequently used fibrinogen reference plasmas and reagents, in the two most popular types of fibrinogen assay: the Clauss and PT-Fg methods, using mechanical and photo-optical coagulometers. The evaluation was performed on behalf of the Medical Devices Agency (MDA), the Department of Health, UK, and the British Committee on Standards in Haematology. Detailed results are available in two MDA reports $(11,12)$.

\section{Materials and Methods}

Reference preparations, assay reagents and coagulometers were selected from those most frequently used in the UK (as judged by UK NEQAS for Blood Coagulation returns). Five centres took part, all experienced in performing coagulation tests. 


\section{Blood Samples}

Blood samples were collected from: healthy normal subjects $(\mathrm{n}=10)$; patients with: DIC $[n=7$, defined by published criteria (13)], liver disease $(n=17)$, congenital hypofibrinogenaemia $(n=4)$, acute phase reaction (APR, 4 with sepsis, 3 severe infection, 1 MI, 1 severe trauma, 1 chronic jaundice); or patients receiving unfractionated heparin therapy $(n=11)$, thrombolytic therapy $(n=10)$, or oral anticoagulants (OAC, INR $>2.5, n=10)$. All blood samples were collected with minimal venous stasis into $0.106 \mathrm{M}$ trisodium citrate. Plasma was prepared by double centrifugation at room temperature, at $2000 \times \mathrm{g}$ for $15 \mathrm{~min}$, to ensure platelet depletion, and stored in aliquots in polypropylene tubes at $-70^{\circ} \mathrm{C}$.

\section{Standards and Reference Plasmas}

The potency of five commercial reference preparations was determined relative to a local working standard (WS), which had been calibrated against the 1st IS for fibrinogen (89/644) (3). WS was prepared by pooling six fresh plasma samples (selected for good optical clarity) from fasting, healthy, normal subjects, and aliquots were rapidly frozen at $-70^{\circ} \mathrm{C}$. The potency of WS was established in three laboratories by Clauss assay, using two commercial reagents ( $\operatorname{Rg} 1$ and $\operatorname{Rg} 2$, see below). These were used on each of two mechanical (Amelung KC4A and KC10, Sigma-Aldrich Diagnostics, Poole, UK) and two photo-optical coagulometers (CA-1000 and CA-6000, Sysmex UK Ltd, Milton Keynes, UK). Two vials each of IS and WS were tested by each method on each of two separate days, with a replicate series of single clotting times for each estimation, obtained in a balanced order. The relative potencies were calculated using parallel line bioassay principles with statistical validation (see below).

The following commercial reference preparations (Rp1-Rp5) were evaluated: Immuno Reference Plasma 100\% (Technoclone Ltd, Dorking, UK), Dade Behring Fibrinogen Standard (Dade Behring, Marburg, Germany), Verify ${ }^{\circledR}$ Reference Plasma (Organon Teknika, Cambridge, UK), Diagen Fibrinogen Standard Plasma (Diagnostic Reagents Ltd, Thame, UK), IL Test ${ }^{\mathrm{TM}}$ Calibration Plasma (Instrumentation Laboratory UK Ltd, Warrington, UK).

Four additional plasmas were used for reference and quality control purposes: a lyophilised high fibrinogen preparation (designated HF, batch 970521), (normal plasma was spiked with 95\% clottable, purified fibrinogen); and three plasmas from non-anticoagulated patients with normal coagulation, having a range of fibrinogen levels: 1-1.5 g/l (QC1), 2-3 g/l (QC2), and 4-5 g/l (QC3). Within run variability was assessed in each method by performing 12 replicate assays (with duplicate clotting times) of the three different QC plasmas. Between run variability was assessed in each method by assaying QC1 with each batch of patient samples.

\section{Assays}

The sensitivity and linearity of the calibration curves obtained for each commercial reference preparation in the modified Clauss assay (14), were assessed over a wide series of dilutions. The potency of each commercial reference preparation was determined in relation to WS using replicate assays tested in a $6 \times 6$ Latin square design, using two Clauss reagents (Rg1 and $\mathrm{Rg} 2$, see below). These were used on photo-optical (1 $\times$ CA-1000 and $2 \times$ CA-6000) and mechanical $(1 \times \mathrm{KC} 4 \mathrm{~A}$ and $2 \times \mathrm{KC10})$ coagulometers amongst 3 participating sites, so that 72 potency estimates of each preparation were obtained. Reference preparations were tested at four dilutions in duplicate (assays accepted with minimum of three dilutions giving valid results).

The performance of four commercial Clauss reagents (Rg1-Rg4): Immuno AG Fibrinogen Reagent (Technoclone), Fibrinogen Determination Reagent (Dade Behring), Fibriquick thrombin reagent (Organon Teknika), and IL Test ${ }^{\mathrm{TM}}$ Fibrinogen-C kit (Instrumentation Laboratory), was assessed using clinical samples. Nine instruments, comprising mechanical $(1 \times \mathrm{KC} 4 \mathrm{~A}$, $2 \times \mathrm{KC10})$ and photo-optical coagulometers [1× CA-1000, $2 \times$ CA-6000, $1 \times$ ACL-300R and 2X ACL-3000 (Sysmex and Instrumentation Laboratory)] were used amongst four different laboratories. The Fibrinogen- $\mathrm{C}$ kit was only used on ACL coagulometers, for which it was specifically designed. A single calibration curve, constructed by taking the mean of ten curves, was used throughout the study for each reagent/instrument combination. Clinical samples were assayed once by each centre, in batches of ten, with duplicate clotting times at a dilution of $1 / 10$. For low fibrinogen levels $(<1.0 \mathrm{~g} / \mathrm{l})$, a $1 / 5$ dilution was used; for high fibrinogen levels ( $>4.0 \mathrm{~g} / \mathrm{l})$, a $1 / 20$ dilution was used. The CA-analysers were set for re-dilution: $<1.0 \mathrm{~g} / \mathrm{l}$ and $>4.0 \mathrm{~g} / \mathrm{l}$.

The commercial reference preparations were assessed by the PT-Fg method, using three ACL and three CA coagulometers (ACL-300/ACL-3000 and CA-1000/CA-6000). An optically clear recombinant thromboplastin, Innovin (Dade Behring) and a relatively turbid rabbit brain thromboplastin, PT Fib HS Plus (PT-FibHS+, Instrumentation Laboratory) were selected for study. The instruments were separately calibrated with each thromboplastin, and each of the following plasmas: WS, HF, Rp1-Rp5. Plasma samples with high, normal, or low fibrinogen levels (QC1, QC2, QC3) were assayed in duplicate in a balanced order, against each of these calibrations. An acid wash was performed between each calibration, to prevent any possible carry-over of reagent. The performance of the PT-Fg methods was also studied, by assaying the 79 clinical samples against WS. These assays were performed in two separate series, where the QC plasmas were tested at the beginning and end of the run to check both for within run precision and temporal drift.

Several other fibrinogen assays were performed for reference purposes. Clottable protein assays (3) were performed by a modified clot extraction method (15). An immuno-nephelometric assay for fibrinogen antigen (Fg:Ag) on the BN-100 analyser, using a commercial latex reagent (Dade Behring), and an ELISA method using polyclonal antisera (Dako Ltd, Ely, UK) were performed, all standardised using WS. D-dimer (Agen Dimertest Gold kit, Quadratech, Surrey, UK) and fibrinogen specific degradation products (FgDP, Organon Teknika) were assayed in the clinical samples by ELISA. The opacity of each reference preparation, QC plasma, and clinical sample was assessed spectrophotometrically using doubling dilutions, at 405, 540, and $650 \mathrm{~nm}$.

\section{Potency Estimation and Statistical Analysis}

The dose-response curve of each fibrinogen reference preparation was checked for linearity and parallelism to the calibrator (i.e. 1st IS or WS). These data were analysed by parallel-line bioassay (16) relating log-transformed dose, as this gave the best linear dose-response curves with homogeneity of variance across the response groups. An analysis of variance provided an assessment of linearity and parallelism and estimates of relative potency were produced from the fitted lines together with $95 \%$ confidence limits. Deviations from linearity and parallelism were considered significant at $\mathrm{p}<0.05$.

For imprecision studies and clinical assays, potency values were obtained from the coagulometer software, or in the case of the semi-automated mechanical end-point systems, by computer software using linear regression analysis. Unless otherwise stated, the arithmetic mean is shown. The distribution of data was examined by measuring skewness and kurtosis, plotting histograms, and where necessary, assessing normality using the Kolmogorov-Smirnov test. Paired t-tests or non-parametric tests (Wilcoxon rank sum paired test) were used as appropriate to the distribution. In regression analysis, outliers were rejected where they were outside the $95 \%$ confidence interval, or where they were clearly separated from the main data cluster (by subjective assessment).

\section{Results}

Calibration curves were generally linear between $1 / 5$ and 1/30 dilutions with IS on the KC-analysers. With WS and the commercial standards (Rp1-5), linearity was usually only obtained on CA-analysers, when the 1/5 dilution was omitted (regardless of the analyser), resulting in calibration curves from $3 / 20$ to $1 / 30$ dilutions. A total of 16 potential calibration values were generated for WS, but very poor parallelism between IS and WS was obtained, with $\mathrm{Rg} 2$ in three estimates on the CA-analysers, even when the extreme dilutions were 
omitted (non-parallel results excluded from analysis). The geometric mean potency of WS was $2.33 \mathrm{~g} / \mathrm{l}(95 \%$ confidence limits $2.28-2.38 \mathrm{~g} / \mathrm{l})$.

An acceptable degree of imprecision was obtained for the three QC plasmas in Clauss assays [intra-assay coefficient of variation (cv) 5.9\%, inter-assay cv 8.5\%]. Greatest variability was observed using the KC-coagulometers (cv 9.9\%). Similar imprecision was obtained for each reagent; $\operatorname{Rg} 4$ gave cv's between 3.29 and $4.07 \%$ on ACL analysers. The overall variability in PT-Fg assays, detected using the QC plasmas, was similar to that for the Clauss assays (pooled intraassay cv 5.7\%). The imprecision tended to be smaller (cv 3-6\%) with the ACL- than with the CA-analysers (cv 8-15\%). This difference was mainly due to high variability with PT-FibHS+ reagent on the CAanalysers, and this reagent also showed higher overall variability than Innovin (cv 6-13\% vs. 6-8\%).

\section{Clauss Fibrinogen Assay}

The geometric mean potency estimate of each commercial reference preparation was calculated from a maximum of 72 assays against WS (Table 1). Non-parallel dose/response lines occurred more often with $\mathrm{Rp} 2$, but were independent of the reagent used. Potency estimates were therefore not available for this preparation in two assay sets (using Rg1 and $\mathrm{Rg} 2$ ), both on CA-analysers. These non-parallel responses were excluded, and potency estimates were analysed from 60 of the 72 assays.

There was generally close agreement between centres and analysers for each reference preparation, with only a small spread of values (Table 1). Overall, there were no significant differences between the values generated in each centre, for any of the commercial reference preparations. There was also no significant difference in mean values between $\operatorname{Rg} 1$ and $\operatorname{Rg} 2$ Clauss reagents (2.55 and $2.61 \mathrm{~g} / \mathrm{l}$ respectively). However, when data for individual standards were analysed, the CAanalysers consistently gave slightly higher values than $\mathrm{KC}$-analysers (overall mean \pm SD $2.66 \pm 0.27$ and $2.51 \pm 0.27 \mathrm{~g} / \mathrm{l}$, respectively).

The estimated potency of two of the commercial reference preparations (Rp1 and Rp2) was in good agreement with the potency declared by the manufacturer (Table 1). Rp4 had a lower Clauss value than the manufacturer's declared potency of $2.50 \mathrm{~g} / \mathrm{l}$, although the latter potency was within the observed range of results and $95 \%$ confidence intervals. Two further commercial preparations (Rp3 and Rp5) showed 11-17\% lower potency values than declared by the manufacturers. The declared potencies of Rp3 and Rp5 did not fall within the range of observed Clauss values. There was closer agreement between clottable protein and Clauss assay results than with the manufacturers' declared potencies. WS contained $2.29 \mathrm{~g} / \mathrm{l}$ fibrinogen by clottable protein assay, compared with the value of $2.33 \mathrm{~g} / \mathrm{l}$ obtained in the Clauss assays.

The mean variability in the results using the 79 clinical samples was generally higher than with the quality control plasmas, particularly in the thrombolytic therapy group, although this was not caused by any particular reagent or instrument. There were no significant differences in mean fibrinogen between the four Clauss reagents (range 2.65 to $2.71 \mathrm{~g} / \mathrm{l})$. However, when the results were divided into different clinical groups (data not shown), the results obtained with $\mathrm{Rg} 1, \mathrm{Rg} 2$, and $\mathrm{Rg} 3$ were generally about $15 \%$ lower than the results with $\operatorname{Rg} 4$ in DIC, and up to $20 \%$ lower in thrombolytic therapy. There were no overall statistically significant differences between centres and no clinically significant differences between analysers (mean $\pm \mathrm{SD}, \mathrm{CA}$-analysers $2.63 \pm 1.84, \mathrm{KC}$-analysers $2.84 \pm 1.86 \mathrm{~g} / \mathrm{l}, \mathrm{p}<0.0001)$. When the results for each Clauss reagent were compared with the mean of the values for the other three reagents, good agreement was obtained over the normal range (maximum mean difference $0.04 \mathrm{~g} / \mathrm{l}$ ). However, the relationship between the results obtained with $\mathrm{Rg} 4$ and other reagents appeared to change with increasing magnitude of fibrinogen concentration. The two samples with the highest fibrinogen concentration gave the greatest scatter in results.

\section{PT-derived Fibrinogen}

It was not possible to calibrate the ACL analysers for PT-Fg assay, using PT-FibHS+ reagent, when reference preparation HF (declared potency $4.65 \mathrm{~g} / \mathrm{l}$ ) was used. The instrument would not accept a calibrant value of $>4.5 \mathrm{~g} / \mathrm{l}$, but attempts to calibrate by entering a nominal value of $4.5 \mathrm{~g} / \mathrm{l}$ were also unsuccessful. This may have been due to short clotting times, or the degree of light scatter being outside set limits. ACL analysers were successfully calibrated with HF using Innovin reagent, when a nominal value of $4.5 \mathrm{~g} / \mathrm{L}$ was entered. $\mathrm{CA}$-analysers could be calibrated with HF provided that higher dilutions were used, to obtain a linear dose response curve. Some calibration failures occurred with Rp2 plasma as calibrant on the ACL, with Innovin reagent. The software failed to report results for QC3 plasma, as the values were outside the set limits for the assay.

The PT-Fg values for the three QC plasmas were invariably higher (regardless of the commercial reference plasma used) than those from Clauss or clottable protein methods. The QC plasma results provided evidence that the choice of calibrator affected the PT-Fg potency estimate (Table 2). Results with WS as calibrant were closest to those from Clauss and clottable protein assays. These were followed by the estimates obtained using Rp1 and HF as calibrants. The most discrepant values were obtained when Rp2 was calibrant. Correction of the results according to the calibrant potency as measured in Clauss assays, failed to remove the discrepancies. The difference in potency assignment was most marked with the high fibrinogen QC sample (QC3), with values varying by more than $2 \mathrm{~g} / \mathrm{l}$.

PT-Fg variability between reagents/analysers was greater in clinical than QC samples, particularly in thrombolytic therapy. Assays using Innovin consistently showed higher values (mean \pm SD $3.56 \pm 2.38$, range 0.52-10.48 g/l) than those using PT-FibHS+ $(3.24 \pm 1.98$, $0.59-7.71 \mathrm{~g} / \mathrm{L}, \mathrm{p}<0.0001)$, although there was good correlation $(\mathrm{r}=0.97)$. Data analysis by clinical subgroup showed no significant differences in mean values for each reagent in: normal subjects, liver disease, or heparin therapy. However, Innovin gave significantly higher PT-Fg values in DIC (mean \pm SD: $1.58 \pm 0.59$ vs. $1.31 \pm 0.52 \mathrm{~g} / \mathrm{l}$, $\mathrm{p}<0.02)$, oral anticoagulation $(4.56 \pm 1.42$ vs. $3.81 \pm 1.11 \mathrm{~g} / \mathrm{l}, \mathrm{p}=0.005)$, thrombolytic therapy $(2.51 \pm 0.73 v s .2 .09 \pm 0.68 \mathrm{~g} / \mathrm{l}, \mathrm{p}<0.01)$, and in

Table 1 Declared and estimated potencies of reference preparations. The declared potency provided by the manufacturer, mean Clauss fibrinogen potency ( $n=60$, range in parenthesis), and clottable protein levels are shown

\begin{tabular}{|l|c|c|c|}
\hline Preparation & $\begin{array}{c}\text { Declared potency } \\
(\mathbf{g} / \mathbf{l})\end{array}$ & $\begin{array}{c}\text { Clauss assay } \\
(\mathbf{g} / \mathbf{l})\end{array}$ & $\begin{array}{c}\text { Clottable protein } \\
(\mathbf{g} / \mathbf{l})\end{array}$ \\
\hline Rp1 & 2.38 & 2.42 & 2.42 \\
\hline Rp2 & 2.54 & $2.32-2.57)$ & 2.84 \\
\hline Rp3 & 3.45 & $(2.37-2.77)$ & 3.09 \\
\hline Rp4 & 2.50 & $2.93-3.25)$ & 2.49 \\
\hline Rp5 & 3.04 & 2.37 & 2.23 \\
\hline
\end{tabular}




\begin{tabular}{|c|c|c|c|c|}
\hline $\begin{array}{c}\text { Calibrant } \\
\text { Plasma }\end{array}$ & $\begin{array}{c}\text { Declared } \\
\text { Potency of } \\
\text { Calibrant ( } g / \mathrm{I})\end{array}$ & $\begin{array}{c}\text { Test plasma } \\
\text { QC1 } \\
\text { (g/l) }\end{array}$ & $\begin{array}{c}\text { Test plasma } \\
\text { QC2 } \\
(\mathrm{g} / \mathrm{l})\end{array}$ & $\begin{array}{c}\text { Test plasma } \\
\text { QC3 } \\
\text { (g/l) }\end{array}$ \\
\hline WS & 2.33 & $2.05(0.20)$ & $2.35(0.20)$ & $5.92(0.68)$ \\
\hline Rp1 & 2.38 & $2.36(0.22)$ & $2.64(0.16)$ & $6.39(0.77)$ \\
\hline Rp2 & 2.54 & $3.69[1.25)$ & $4.09(0.97)$ & $8.51(1.70)^{4}$ \\
\hline Rp3 & 3.45 & $2.90(0.44)$ & $3.48(0.87)$ & $7.78(1.35)$ \\
\hline Rp4 & 2.50 & $2.87\{0.47\rangle$ & $3.22(0.29)$ & $7.76(1.25)$ \\
\hline Rp5 & 3.04 & $2.74\{0.20\rangle$ & $2.07(0.19)$ & $7.71(1.02)$ \\
\hline $\mathrm{HF}$ & 4.50 & $2.63^{*}(0.73)$ & $2.89^{*}(0.61)$ & $7.03^{*}(2.31)$ \\
\hline \multicolumn{5}{|c|}{ Mean values $(\mathrm{g} / \mathrm{l})$ from other methods } \\
\hline \multicolumn{2}{|l|}{ Clauss } & 1.93 & 2.40 & 4.82 \\
\hline \multicolumn{2}{|c|}{ Clottable protein } & 1,90 & 2.22 & 4.54 \\
\hline
\end{tabular}

Table 2 PT-Fg assays (combined results from all laboratories, reagents and analysers) of three quality control plasmas using different reference plasmas as calibrants. Mean potencies are shown, with the SD in parenthesis $(n=12)$. The Declared Calibrant Potency is that declared by the manufacturer, or in the case of WS, the value obtained by Clauss assay. * Values exclude data from ACL-analysers with IL PT-FibHS+, where calibration failed; $* * n=9$ (calibration failed with Rp2 and Innovin on the ACL)

\begin{tabular}{|c|c|c|c|c|c|c|c|}
\hline & $\begin{array}{l}\text { ACL- } \\
300 R\end{array}$ & $\begin{array}{l}\text { ACL- } \\
3000\end{array}$ & $\begin{array}{c}\text { All } \mathrm{ACL} \\
\text { analysers }\end{array}$ & $\begin{array}{l}\text { CA- } \\
1000\end{array}$ & $\begin{array}{l}\text { CA- } \\
6000\end{array}$ & $\begin{array}{c}\text { All CA } \\
\text { analysers }\end{array}$ & $\begin{array}{c}\text { All } \\
\text { analysers }\end{array}$ \\
\hline Mean & 3.52 & $3.41^{*}$ & 3.46 & 3.13 & $3.44^{* *}$ & $3.29^{*+4}$ & 3.37 \\
\hline Min & 0.49 & 0.67 & 0.49 & 0.75 & 0.70 & 0.70 & 0.49 \\
\hline $\operatorname{Max}$ & 9.92 & 8.29 & 9.82 & 8.30 & 9.57 & 9.57 & 9.92 \\
\hline SD & 2.49 & 2.16 & 2,31 & 1.92 & 2.24 & 2.04 & 2.17 \\
\hline
\end{tabular}

Table 3 Effect of coagulation analyser on PT-Fg assays $(\mathrm{g} / \mathrm{l})$ in clinical samples

the "high fibrinogen" group (7.27 \pm 1.06 vs. $6.16 \pm 0.92 \mathrm{~g} / \mathrm{l}, \mathrm{p}<0.01)$. Hypofibrinogenaemia samples gave values of: 1.40, 0.52, 1.27, and $1.09 \mathrm{~g} / \mathrm{l}$ for Innovin and 1.19, 1.28, 1.06, and $1.79 \mathrm{~g} / \mathrm{l}$ for PT-FibHS+, although there was great variability in the results. Clear discrepancies in the results with these two reagents were also observed in other samples; differences of $0.5-1.0 \mathrm{~g} / \mathrm{l}$ were seen in samples with low or normal fibrinogen levels, and 2.0-3.0 g/l in high fibrinogen samples.

Significantly higher PT-Fg levels were measured on ACL-analysers compared to CA-analysers (Table 3). There were no significant differences between different ACL analyser models, but the CA-6000 gave higher values (similar to the ACL analysers) than the CA-1000. Higher levels were obtained with ACL analysers for thrombolytic therapy (mean \pm SD $2.40 \pm 0.74$ vs. $2.07 \pm 0.64 \mathrm{~g} / \mathrm{l}, \mathrm{p}<0.001$ ), oral anticoagulant ( $4.29 \pm 1.12$ vs. $3.88 \pm 1.18 \mathrm{~g} / \mathrm{l}, \mathrm{p}=0.023)$, and "high fibrinogen" groups $(7.38 \pm 1.19$ vs. $6.26 \pm 1.04 \mathrm{~g} / \mathrm{l}, \mathrm{p}<0.001)$. Hypofibrinogenaemia patients gave higher values by the CA-analysers $(1.24 \pm 0.12$ vs. $1.01 \pm 0.24 \mathrm{~g} / \mathrm{l}, \mathrm{p}=0.028)$.

\section{Comparison of Clauss and PT-derived Fibrinogen}

PT-Fg was higher than Clauss fibrinogen $(n=79$, mean \pm SD: 3.39 \pm 2.16 and $2.68 \pm 1.82 \mathrm{~g} / \mathrm{l}, \mathrm{p}<0.0001$, respectively) in all clinical groups, except for the four hypofibrinogenaemia patients $(1.21 \pm 0.22$

Table 4 Absorption values of 1/8 diluted reference plasmas at three wavelengths nb. All preparations had been lyophilised with the exception of WS

\begin{tabular}{|l|l|l|l|}
\hline & A405 & A540 & A650 \\
\hline Rp1 & 0.637 & 0.372 & 0.28 \\
\hline Rp2 & 0.988 & 0.602 & 0.451 \\
\hline Rp3 & 0.473 & 0.265 & 0.196 \\
\hline Rp4 & 0.672 & 0.398 & 0.300 \\
\hline Rp5 & 0.581 & 0.371 & 0.259 \\
\hline HF & 0.623 & 0.379 & 0.286 \\
\hline W5 & 0.209 & 0.074 & 0.043 \\
\hline IS & 0.362 & 0.202 & 0.153 \\
\hline
\end{tabular}

and $1.20 \pm 0.16)$ and the normal subjects $(2.16 \pm 0.54$ and $2.10 \pm 0.41)$. There was an apparent correlation $(r=0.95)$ between the two types of assay, but the best fit line was sigmoid (Fig. 1a). The thrombolytic therapy samples clearly distorted this relationship, but a sigmoid curve fit was still obtained after their exclusion. A similar type of curve fit applied to both thromboplastins (Fig. 1b), although there was less scatter with PT-FibHS+ than Innovin. The curve reached a plateau earlier with PT-FibHS+, giving a lower upper limit of measurement for PT-Fg (approximately $7 \mathrm{~g} / \mathrm{l}$, compared to $9 \mathrm{~g} / \mathrm{l}$ with Innovin and equivalent to $5.0-6.0 \mathrm{~g} / \mathrm{l}$ by Clauss assay).

\section{Plasma Opacity Analysis}

The absorbance of doubling dilutions of each reference preparation was measured in a spectrophotometer at three wavelengths $(405,540$, $650 \mathrm{~nm}$ ), selected to cover different regions of the visible spectrum. One of these wavelengths was similar to that used by the photo-optical coagulometers when coagulation tests are performed $(660 \mathrm{~nm})$. The reference preparations showed a wide range of absorbance values (Table 4). Rp2 gave the highest absorbance at each wavelength, even after 1/8 dilution, while WS had relatively low absorbance, in keeping with its apparent optical clarity. The degree of discrepancy between Clauss and PT-Fg values appeared to correlate (Fig. 2) with the absorbance of the PT-Fg calibration plasma $(r=0.83, p=0.02$ and $r=0.76$, $\mathrm{p}<0.05$, for QC1 and QC3 at $650 \mathrm{~nm}$ ). Similar results were obtained at 405 and $540 \mathrm{~nm}$. The most turbid reference plasma (Rp2) gave the greatest difference between assays, whereas the least turbid (WS) gave the lowest discrepancy. A wide range of plasma opacities existed in the clinical samples (e.g. range of absorbance values obtained for $1 / 5$ diluted samples at $405 \mathrm{~nm}$ : 0.137-2.680; $540 \mathrm{~nm}$ : 0.040-1.917, $650 \mathrm{~nm}$ : $0.022-1.410)$. In some samples, a considerable portion of this was related to icterus and/or lipaemia. When all of these samples were analysed as a group, there was no obvious relationship between plasma opacity and the discrepancy between Clauss and PT-Fg assays. However, analysis of the normal control and oral anticoagulant samples (to remove the effects of abnormal fibrinogen molecules and liver dysfunction) showed a significant correlation between absorbance and the discrepancy between PT-Fg and Clauss assays (405 nm: $r=0.50$, 
$\mathrm{p}=0.025 ; 540 \mathrm{~nm}: \mathrm{r}=0.55, \mathrm{p}=0.013 ; 650 \mathrm{~nm}: \mathrm{r}=0.56, \mathrm{p}=0.01) . A$ similar correlation was obtained with PT-Fg, but not with Clauss or $\mathrm{Fg}: \mathrm{Ag}$ assays (data not shown).

\section{Fibrinogen Antigen Assays}

Fg:Ag was measured by ELISA in 79 samples (mean \pm SD: $3.19 \pm$ $2.08 \mathrm{~g} / \mathrm{l}$ ). There was sufficient plasma in 68 samples, to compare both Fg:Ag assays. These showed no significant difference, although there was a trend towards lower levels by the immuno-nephelometric method than ELISA (mean \pm SD: $3.02 \pm 1.63$ and $3.25 \pm 2.10 \mathrm{~g} / \mathrm{l}$, respectively). A good correlation $(r=0.92, p<0.0001, y=0.71 X+0.71)$ was obtained, although there was a high degree of scatter. Fg:Ag assays gave significantly higher values than Clauss assays (mean \pm SD: $2.92 \pm$ $1.86 \mathrm{~g} / \mathrm{l}, \mathrm{p}<0.0001$ and $\mathrm{p}<0.003$ ), but slightly lower values than PT-Fg assays $(3.72 \pm 2.16 \mathrm{~g} / \mathrm{l}, \mathrm{p}<0.001$ and $\mathrm{p}=0.026)$. A good correlation was obtained between Fg:Ag, and either Clauss $(r=0.86, p<0.0001)$ or PT-Fg assays $(r=0.88, p<0.0001)$ although there was a high degree of scatter (data not shown). Closer agreement was obtained between $\mathrm{Fg}: \mathrm{Ag}$ and PT-Fg than Clauss fibrinogen. In patients with elevated fibrinogen levels $(>4.5 \mathrm{~g} / \mathrm{l})$, there were large discrepancies between
Fig. 1a Influence of clinical group on the relationship between PT-Fg and Clauss fibrinogen assays. Results are shown for disseminated intravascular coagulation $(\boldsymbol{\square})$, liver disease $(\triangle)$, congenital hypofibrinogenaemia $(\bigcirc)$, heparin therapy $(\mathbf{+})$, thrombolytic therapy $(\mathbf{\Delta})$, oral anticoagulants $(\mathbf{O})$, acute phase reaction $(\boldsymbol{\nabla})$, normal controls $(\diamond)$ and quality control samples $(\square)$. The line of best fit is shown for all data (solid line) and after excluding the thrombolytic therapy samples (broken line).
Fig. $1 b$ Comparison of PT-Fg values obtained with Innovin $(-)$ or PT-FibHS+ $(\triangle)$ and mean Clauss fibrinogen
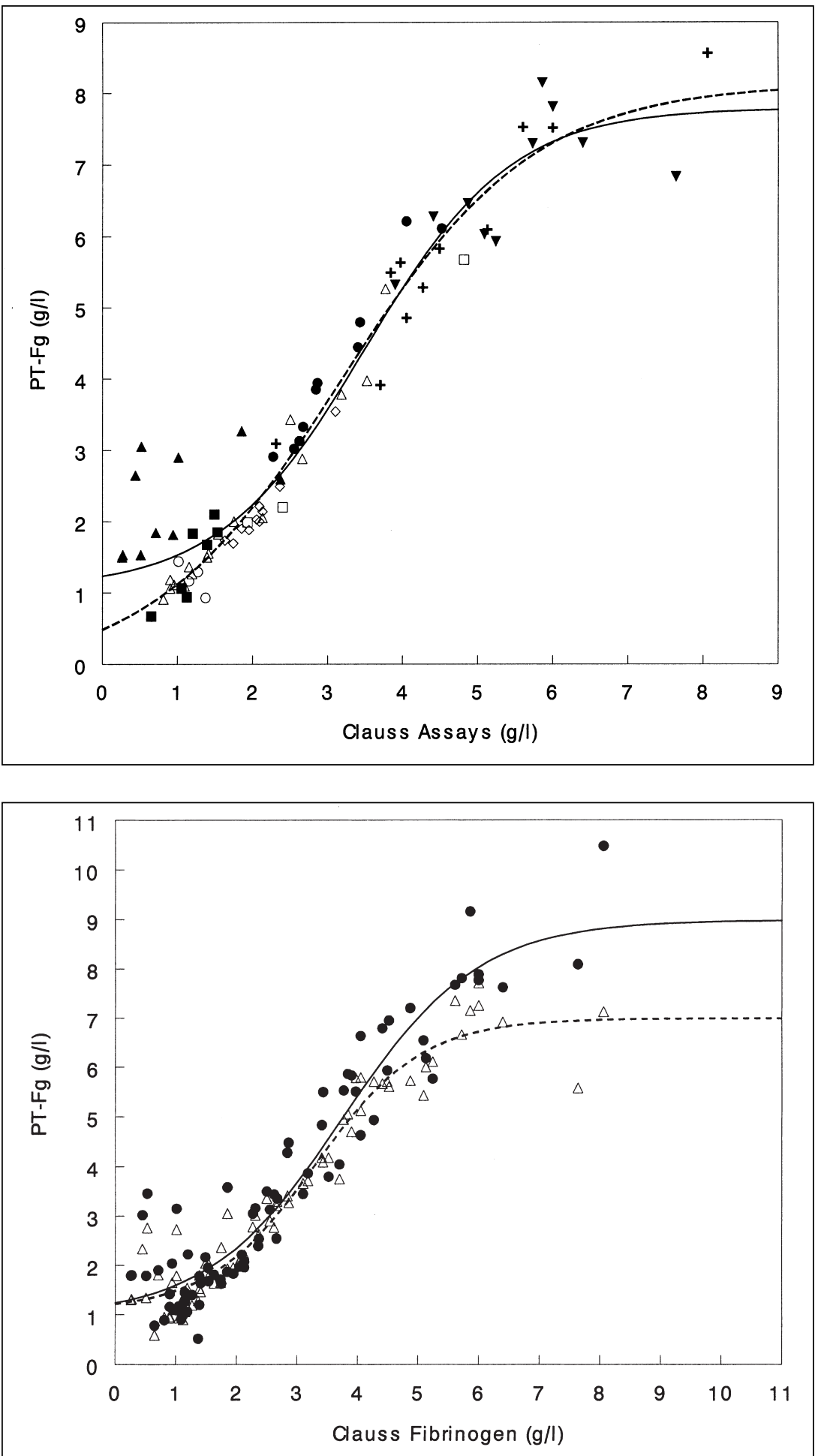


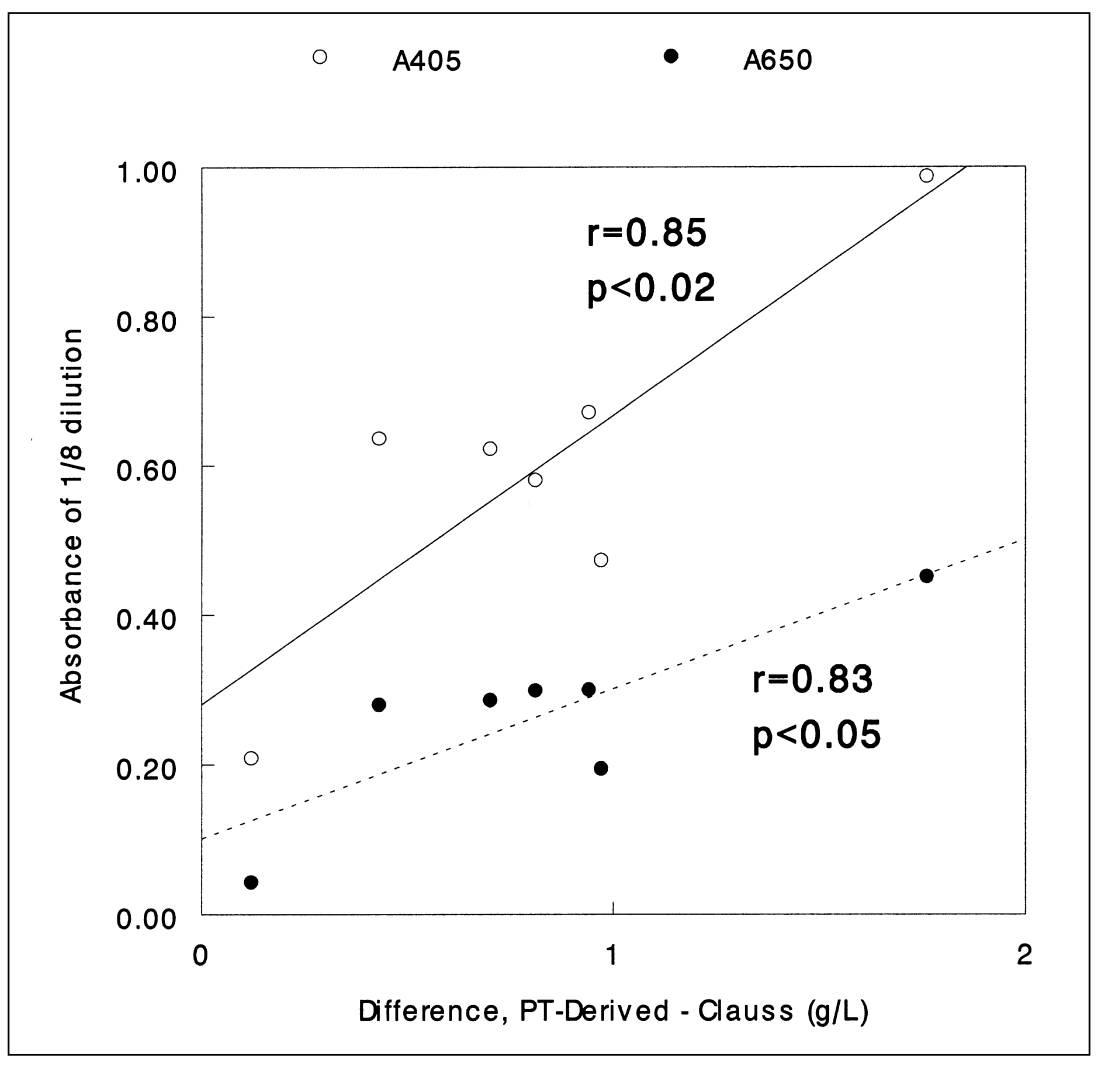

Fig. 2 The relationship between calibrant plasma absorbance and discrepancy between PT-Fg and Clauss assays. The difference between PT-Fg and Clauss assay values obtained for $\mathrm{QC1}$ plasma, when assayed against each calibrant, was plotted against the absorbance (at 405 and $650 \mathrm{~nm}$ ) of a 1/8 dilution of each calibrant plasma

either Clauss or PT-Fg assays and Fg:Ag, and there was some variation depending on the type of clinical problem.

\section{Influence of Fibrin Degradation on Fibrinogen Assay}

The levels of D-dimer and FgDp (range 15-6439 ng/ml and 220-18,000 ng FEU/ml, respectively, excluding the thrombolytic therapy samples), showed no correlation with the fibrinogen levels in each assay type, or with the discrepancy between Clauss and PT-Fg results. D-dimer levels were not raised in the majority of thrombolytic therapy samples (median 131, range 32-1751 ng/ml), but FgDP levels were abnormally high in all except one (median 18,000, range 220-21,380 ng FEU/ml), and were associated with a large discrepancy between the Clauss and PT-Fg assays. When this inter-assay discre-

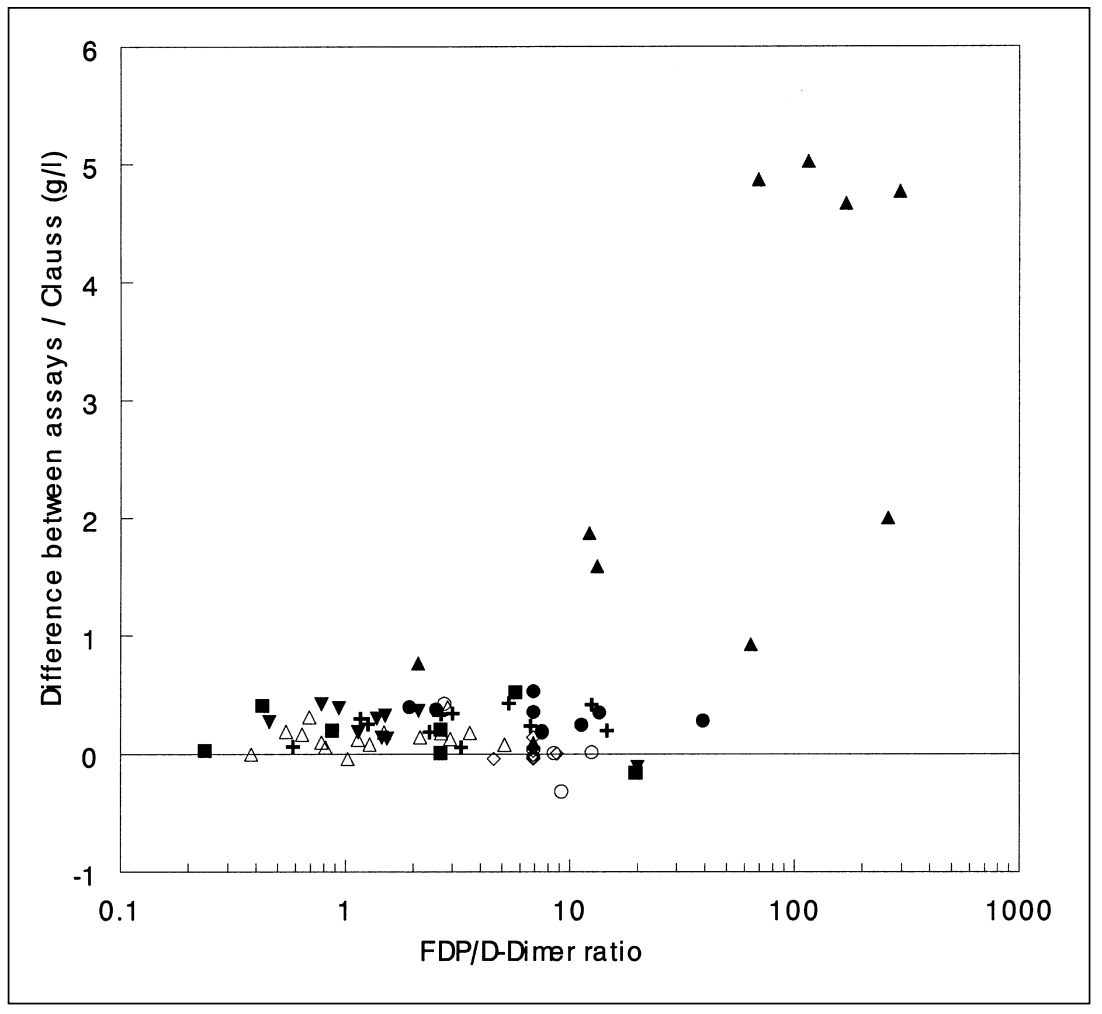

Fig. 3 The influence of degraded fibrinogen on PT-Fg and Clauss assays. The difference between PT-Fg and Clauss assays, as a function of Clauss fibrinogen concentration, was plotted against the ratio of fibrinogen degradation products (FDP) to crosslinked fibrin degradation products. For key, see Fig. 1a 
pancy was weighted according to the Clauss value, and plotted against the ratio of FgDP/D-dimer, the thrombolytic therapy group were clearly separated from other groups, having a high difference per gram of Clauss fibrinogen (Fig. 3).

\section{Discussion}

The use of fibrinogen assays in the investigation of haemorrhage is well established. Prospective studies indicate that fibrinogen is a predictor of cardiovascular events in arterial disease (27); this statistical association remains, regardless of the fibrinogen assay type, although immunoassays could give better prediction than Clauss assays $(28,29)$. Fibrinogen levels may also be increased due to physiological changes, oral contraceptives, and acute phase reaction (30-33), and the contribution to thromboembolism in these situations remains unclear. The measurement of fibrinogen in risk factor screening requires standardised and reproducible assays, which can be universally interpreted. A great variety of methods, reagents and analysers are used for fibrinogen assay $(17,18)$, and laboratories focus on ease of use, throughput and cost. The widespread availability of the PT-Fg $(9,19-25)$ has raised issues about the variability and clinical utility of different fibrinogen assays $(2,5-8,26)$. Most studies suggest disparity between PT-Fg and Clauss assays, but have been limited to particular reagent/analyser combinations; there is little information concerning reagent, analyser and calibrant related discrepancies within each method. We investigated these variables in a multi-centre study. For logistical reasons and to overcome turbidity problems associated with the 1st IS, an optically clear working standard (WS) was prepared. This was used as the main reference preparation in subsequent studies.

The dilution range yielding linear calibration curves for normal plasma by Clauss assay varied depending on the analyser, due to sensitivity differences and accuracy of clot detection. There was an acceptable degree of imprecision and agreement for all Clauss assays with no significant differences between centres or analysers. The only Clauss reagent related discrepancies were seen with $\mathrm{Rg} 4$ (only used on ACL analysers, for which it was optimised), which gave higher values in DIC and thrombolytic therapy. This reagent had a relatively low thrombin concentration $(35 \mathrm{u} / \mathrm{ml})$, and contained calcium ions, which may have improved clot detection with abnormal fibrinogen molecules. These differences would not cause clinically significant problems in routine practice, provided that only a single Clauss fibrinogen method was in use. Reference plasma Rp2 exhibited a number of non-parallel dose response curves, which may have been related to its increased turbidity, since plasmas with low turbidity as judged by photometry gave no calibration problems.

Two reference plasmas (Rp3 and Rp5) had mean Clauss values lower than the declared potency, and this was of clinical significance. The Clauss assays generally showed good agreement with clottable protein assays. However, this type of problem (34) has improved, since a previous report (35) suggested that commercial standards deviated from their declared value by as much as $80 \%$, using clottable protein assays.

It was not possible to calibrate the ACL-analysers for PT-Fg when the high fibrinogen plasma and rabbit brain thromboplastin were used, even when nominal, low fibrinogen values were entered. This problem appeared to involve both reagent turbidity (7) as well as programming limitations of the analysers. A similar problem did not occur on the CA-analysers, although the manufacturers do advise calibration with fresh, optically clear plasma. PT-Fg values were invariably higher than those obtained by Clauss or clottable protein methods, regardless of the commercial reference plasma used, but the PT-Fg potency estimates were closest to the Clauss values when WS was used as calibrant. The largest PT-Fg estimates were obtained using the plasma with the highest relative turbidity. Since both types of analyser utilise a light scattering principle for clot detection, with a wavelength of approximately $660 \mathrm{~nm}$, it is not surprising that turbid plasmas influence their performance, particularly when they have high absorbance in that spectral region. Correction of the results to allow for the differences between the manufacturer's and the estimated potencies in Clauss assays, did not remove the discrepancies in PT-Fg potency. The discrepancy between calibrants was greatest when a high fibrinogen plasma was studied, with values varying by more than $2 \mathrm{~g} / \mathrm{l}$. The differences in potency estimates are likely to be due to the calibration procedures used by the manufacturer. The use of the 2 nd IS (98/612) for potency assignment may help to resolve the problem, since this standard has been prepared by detergent treatment of plasma, has high solubility and good optical clarity, which reduces any turbidity problems (36). In normal and oral anticoagulant test samples, absorbance was correlated with the degree of discrepancy in PT-Fg assays. This was not observed when all clinical samples were studied, probably due to the effects of confounding variables such as: abnormal fibrinogen molecules, lipaemia and icterus.

Differences in PT-Fg values were observed between the CA-1000 and CA-6000, as well as between CA- and ACL-analysers. Contributory factors may be the use of different cuvette types and the calibration method for CA-analysers, based on a single measured point and a hypothetical zero, with the assumption that a linear relationship exists between concentration and light scatter. The PT-Fg assays were influenced by the thromboplastin reagent, and the degree of difference could have caused errors in the clinical management of patients with decreased or low normal fibrinogen levels, while patients with increased fibrinogen showed differences as high as $3 \mathrm{~g} / \mathrm{l}$. This may also be related to the large turbidity differences between the thromboplastin reagents (7), which will influence basal light scatter on the analysers. A small change in light scatter with the optically clear Innovin would be associated with a greater change in fibrinogen concentration than would have occurred with the turbid PT-FibHS+.

The great disparity between PT-Fg and Clauss assays in thrombolytic therapy is well recognised $(5,9,25)$, and suggests that the PT-Fg assay is influenced by partially clottable fibrinogen. The discrepancy between the two assays was associated with FgDP, but not D-dimer concentration. Since FgDP retain at least one fibrinopeptide A domain (37), they are capable of joining fibrin strands, but cause polymer termination. This may delay clot detection in the Clauss technique, although a large opacity change may still be achieved, giving a higher PT-Fg level. Such an effect would become accentuated as the fibrinogen level fell, i.e. in the diluted plasma of a Clauss assay. This could contribute to relatively low Clauss values and higher PT-Fg fibrinogen values, whereas Fg:Ag levels may be unaffected. In fact, the addition of early fibrinogen degradation products has been shown (38) to cause a dose-dependent increase in Clauss and PT-Fg assays, whereas intermediate and late products increase PT-Fg, but decrease Clauss results. More turbid fibrin gels are formed at lower thrombin concentrations (10). The thrombin concentration generated in the PT-Fg method is substantially lower (on a U/ml basis) than that used in the Clauss assay, while the fibrinogen concentration is 2.5- to 5-fold higher, and this may influence the results under conditions of abnormal fibrin polymerisation.

Results from the Clauss and PT-Fg assays followed a sigmoid relationship. This may partly be explained by PT-Fg methods having upper 
cut-off limits, which vary depending on the analyser, while at lower fibrinogen levels there is a sensitivity problem, since the degree of change in light scatter is small. This makes it difficult to apply data correction algorithms to make the assays equivalent, as some have suggested. The PT-Fg measuring range of the thromboplastin reagents differed, due to variability in the fibrinogen level at which a plateau in the sigmoid curve was reached. It remains to be established whether this effect is constant for different batches of the same reagents, and is a general phenomenon with all thromboplastins.

No significant differences were observed between the two Fg:Ag assays, which showed a good correlation, despite a relatively high degree of scatter. The latter is likely to be due to differences in epitope specificity of the antibodies (e.g. the polyclonal antibodies in the ELISA assay cross-react with FDPs), and the binding affinities, resulting in differences in sensitivity and precision (39). Fg:Ag levels were generally higher than Clauss fibrinogen levels, but slightly lower than the PT-Fg values. The discrepancy between Fg:Ag and Clauss values was expected, since the former also measures non-functional and some degraded forms of fibrinogen.

The large number of assays and the limited volumes of clinical samples meant that only single batches of each reference plasma and reagent could be studied and it is possible that some of the discrepancies may be batch-dependent. We understand that Rp2 is no longer commercially available, but the results have demonstrated that reference preparations with high turbidity may cause calibration problems in both Clauss and PT-Fg assays.

In conclusion, there were clinically significant discrepancies between observed and declared potencies for some commercial reference plasmas, and these were amplified in PT-Fg assays. The optical clarity of the calibrant plasma had a significant impact on Clauss and PT-Fg assays. PT-Fg levels varied according to the thromboplastin reagent and analyser used, and there was a sigmoid relationship with Clauss assays. The PT-Fg and Fg:Ag assays yielded similar values in many samples, and PT-Fg assays were not sensitive to FgDP, and may not therefore reflect the true clinical situation. Clinical laboratories should be aware of these discrepancies when selecting fibrinogen assay methods for local use. Pt-Fg assays may be less reliable than Clauss in the investigation of bleeding diathesis, and may be inaccurate in samples with high fibrinogen. It is essential that individual laboratories and multi-centre studies standardise to a particular fibrinogen assay method.

\section{Role of each Author}

The study was conceived by a Working Party of BCSH, comprising: I. J. Mackie, S. Kitchen, G. D. O. Lowe, D. Howarth, and P. J. Gaffney. This group also designed the study with the help of A. S. Lawrie. The assays were performed by: S. Kitchen, A. S. Lawrie, G. Purdy, J. Martin, and A. Rumley, while P. Rigsby provided statistical advice. All of the above contributed to data analysis and manuscript preparation.

\section{Acknowledgements}

The authors would like to thank the staff at each centre for their assistance, Dr. Chris Ludlam and Dr. John Pasi, for providing clinical samples; and Dr. Dati (Behring Diagnostics, Marburg) for plasma HF. We would also like to thank the various manufacturers for providing reagents and reference preparations.

\section{References}

1. Clauss A. Gerinnungsphysiologische Schnellmethode zur Bestimmung des Fibrinogens. Acta Haematol 1957; 17: 237-46.

2. Chantarangkul V, Tripodi A, Mannucci PM. Results of a collaborative study for fibrinogen measurement. Evidence that the use of a common calibrator improves interlaboratory agreement. Blood Coagul Fibrinolysis 1994; 5: 761-6.

3. Gaffney PJ, Wong MY. Collaborative study of a proposed International Standard for plasma fibrinogen measurement. Thromb Haemost 1992; 68: 428-32.

4. U.K. National External Quality Assessment Scheme (NEQAS) for Blood Coagulation, Report on Survey 120, August 2000.

5. Palareti G, Maccaferri M, Manotti C, Tripodi A, Chantarangkul V, Rodeghiero F, Ruggeri M, Mannucci PM. Fibrinogen assays: A collaborative study of six different methods. C.I.S.M.E.L Comitaro Italiano per la Standardizzione dei Methodi in Ematologia e Labatorio 1991; 37: 714-9.

6. Chitolie A, Mackie IJ, Grant D, Hamilton JL, Machin SJ. Inaccuracy of the 'derive' fibrinogen measurement. Blood Coagul Fibrinolysis 1994; 5: 955-7.

7. Chitolie A, Mackie IJ, Machin SJ. The type of thromboplastin reagent has important effects on the PT-derived fibrinogen potency. Laboratory Hematology 1998; 4: 149-55.

8. Lawrie AS, McDonald SJ, Purdy G, Mackie IJ, Machin SJ. Prothrombin time derived fibrinogen determination on the Sysmex CA- $6000^{\mathrm{TM}}$. J Clin Pathol 1998; 51: 462-6.

9. Rossi E, Mondonico P, Lombardi A, Preda L. Method for determination of functional (clottable) fibrinogen by the new family of ACL coagulometers. Thromb Res 1988; 52: 453-68.

10. de Cristofaro R, Landolfi R. Measurement of plasma fibrinogen concentration by the prothrombin-time-derived method: applicability and limitations. Blood Coagul Fibrinolysis 1998; 9: 251-9.

11. Medical Devices Agency Evaluation Report: Fibrinogen Standards and Reference preparations. MDA/99/47, Her Majesty's Stationery Office, Norwich, UK, 1999.

12. Medical Devices Agency Evaluation Report: Fibrinogen Assay Reagents and Methods. MDA/2000/01, Her Majesty's Stationery Office, Norwich, UK, 2000.

13. Kitchen S, Malia RG, Preston FE. A comparison of methods for the measurement of activated factor VII. Thromb Haemost 1992; 68: 301-5.

14. Chanarin I. Laboratory Haematology. An account of laboratory techniques. Churchill Livingstone, Edinburgh, 1989; 279-80.

15. Jacobsson K. Studies in the determination of fibrinogen in human blood plasma. Scan J Clin Lab Invest 1955; 7 (Suppl. 14): 1-54.

16. Finney DJ. Statistical methods in Biological Assays. Third Edition, Charles Griffin and Company Ltd, 1978.

17. Rampling MW, Gaffney PJ. Measurement of fibrinogen in plasma. In: Prog Chemical Fibrinolysis and Thrombosis. Davidson JF, Samama MM, Desnoyers PC, eds. Raven Press, New York, 1976, Vol. 2, pp 91-105.

18. de Maat MPM, Lowe GDO, Haverkate F. Fibrinogen, In: Laboratory Techniques in Thrombosis - A Manual. Jespersen J, Bertina RM, Haverkate F, eds. Kluwer Academic Publishers, Dordrecht, The Netherlands, 1999, pp 79-88.

19. Vermylen C, de Vreker RA, Verstraete M. A rapid enzymatic method for assay of fibrinogen fibrin polymerization time (FPT test). Clin Chim Acta 1963; 8: 418-24.

20. Natelson EA, Dooley DF. Rapid determination of fibrinogen by thrombokinetics. Am J Clin Pathol 1974; 61: 828-33.

21. Becker U, Bartl K, Wahlefeld AW. A functional photometric assay for plasma fibrinogen. Thromb Res 1984; 35: 475-84.

22. Chantarangkul V, Tripodi A, Mannucci PM. Evaluation of a fully automated centrifugal analyzer for performance of hemostasis tests. Clin Chem 1987; 33: 1888-90. 
23. De Metz M, van Wersch JWJ. Use of a centrifugal analyzer for a chromogenic prothrombin time, a chromogenic activated partial thromboplastin time and a kinetic fibrinogen assay in a routine hospital laboratory. Haemostasis 1987; 17: 254-9.

24. Cambas JP, Bierme R, Martinon JC, Dousset B. Evaluation des performances d'un automate en coagulation: l'Electra 700. Nouv Rev Fr Hematol 1985; 25: 313-20.

25. Hoffmann JJML, Verhappen MAL. Automated nephelometry of fibrinogen: analytical performance and observations during thrombolytic therapy. Clin Chem 1988; 34: 2135-40.

26. Kitchen S, Jennings I, Preston FE. Comparison of fibrinogen determinations using a Clauss assay and two prothrombin time derived methods. Thromb Haemost 1995; 73: 1245 (A).

27. Lowe GDO, Rumley A. Use of fibrinogen and fibrin D-dimer in prediction of arterial thrombotic events. Thromb Haemost 1999; 82: 667-72.

28. Cremer P, Nagel D, Labrot B, Mann H, Muche R, Elster H, Seidel C. Lipoprotein $\mathrm{Lp}(\mathrm{a})$ as predictor of myocardial infarction in comparison to fibrinogen, LDL cholesterol and other risk factors: results from the prospective Göttingen Risk Incidence and Prevalence Study (GRIPS). Eur J Clin Invest 1994; 24: 444-53.

29. Sweetnam PM, Yarnell JWG, Lowe GDO, Baker IA, O'Brien JR, Rumley A, Etherington MD, Whitehead PJ, Elwood PC. The relative predictive power of heat-precipitation nephelometric and clottable (Clauss) fibrinogen in the prediction of ischaemic heart disease: the Caerphilly and Speedwell studies. Br J Haematol 1998; 100: 582-8.

30. Hantgan RR, Francis CW, Marder VJ. Fibrinogen structure and physiology. In: Hemostasis and Thrombosis: Basic Principles and Clinical Practice. Colman RW, Hirsh J, Marder VJ, Salzman EW, eds. JB Lippincott Co., Philadelphia, 1994; 277-300.

31. Lowe GDO, Rumley A, Woodward M, Morrison CE, Philippou H, Lane DA, Tunstall-Pedoe H. Epidemiology of coagulation factors, inhibitors and activation markers: The third Glasgow MONICA survey. I) Illustrative reference ranges by age, sex and hormone use. Br J Haematol 1997; 97 : 775-84.

32. Kluft C, Lansink M. Effect of oral contraceptives on haemostasis variables. Thromb Haemost 1997; 78: 315-26.

33. Humphries SE, Henry JA, Montgomery HE. Gene-environment interaction in the determination of levels of haemostatic variables involved in thrombosis and fibrinolysis. Blood Coagul Fibrinolysis 1999; 10 (Suppl 1): S17-S21

34. Halbmayer WM, Haushofer A, Schon R, Radek J, Fischer M. Comparison of a new automated kinetically determined fibrinogen assay with the 3 most used fibrinogen assays (functional, derived and nephelometric) in Austrian laboratories in several clinical populations and healthy controls. Haemostasis $1995 ; 25$ : 114-23.

35. Furlan M, Felix R, Escher N, Lammle B. How high is the true fibrinogen content of fibrinogen standards? Thromb Res 1989; 56: 583-92.

36. Whitton CM, Sands D, Hubbard AR, Gaffney PJ. A collaborative study to establish the 2 nd international standard for fibrinogen, plasma. Thromb Haemost 2000; 84: 258-62.

37. Nieuwenhuizen W, Bos R. Soluble fibrin and degradation products of fibrinogen (FgDP), fibrin (FbDP; D-dimer) and total of FgDP and FbDP (TDP). In: Laboratory Techniques in Thrombosis - A Manual. Jespersen J, Bertina RM, Haverkate F, eds. Kluwer Academic Publishers, Dordrecht, The Netherlands, 1999; 275-84.

38. Oosting JD, Hoffmann JJML. Evaluation of an automated photometric fibrinogen assay. Blood Coagul Fibrinolysis 1997; 8: 321-6.

39. Hoffman M, Greenberg CS. The effects of fibrin polymerization inhibitors on quantitative measurements of plasma fibrinogen. Am J Clin Pathol 1987; 88: 490-3.

Received June 30, 2001 Accepted after resubmission February 28, 2002

\section{Ordering has never been so easy:} http://www.schattaver.com 